\title{
Identificación de necesidades de formación continua del profesorado ante las demandas educativas del siglo XXI
}

Identification of continuing training needs for teachers in the face of 21st century educational demands

\section{Volumen 21, Número 1 \\ Enero - Abril \\ pp. 1-32}

\author{
Andrés Rodríguez Jiménez \\ Bertha Miqueli Rodríguez \\ Yosdey Dávila Valdés
}

\section{Citar este documento según modelo APA}

Rodríguez Jiménez, Andrés., Miqueli Rodríguez, Bertha. y Dávila Valdés, Yosldey. (2021). Identificación de necesidades de formación continua del profesorado ante las demandas educativas del siglo XXI. Revista Actualidades Investigativas en Educación, 21(1), 1-32. Doi. 10.15517/aie.v21i1.44073 


\title{
Identificación de necesidades de formación continua del profesorado ante las demandas educativas del siglo XXI
}

Identification of continuing training needs for teachers in the face of 21 st century educational demands

\author{
Andrés Rodríguez Jiménez ${ }^{1}$ \\ Bertha Miqueli Rodríguez ${ }^{2}$ \\ Yosdey Dávila Valdés ${ }^{3}$
}

\begin{abstract}
Resumen: La propuesta de un modelo pedagógico que dé respuesta a las demandas educativas del siglo XXI en la Universidad de Artemisa, Cuba, requirió de la identificación de necesidades formativas del profesorado para su implementación, hecho que justificó esta investigación y constituyó su objetivo. El estudio se realizó en 2018 con el empleo de una metodología esencialmente cuantitativa mediante una encuesta que incluyó preguntas cerradas y abiertas, así como una escala tipo Likert. Los resultados indicaron que, aunque no en todos los casos, las personas encuestadas fueron suficientemente reflexivas para reconocer en si mismas las carencias formativas, estas carencias estuvieron presentes. Por otra parte, se evidenciaron limitaciones en el trabajo metodológico como actividad formativa esencial, y el personal docente encuestado identificó la falta de una oferta de formación continua por parte de la Universidad. Los resultados representaron tan solo la primera etapa del proceso de identificación de necesidades formativas, pues estos resultados deben enriquecerse con: las políticas universitarias para el período electivo, los informes de resultados del trabajo metodológico en la institución, así como con los resultados de las evaluaciones anuales y de los controles a clases realizados al profesorado. Además, como todo proceso, deberá alimentarse y retroalimentarse con cierta regularidad para que su ciclo se actualice de manera permanente. En cuanto a la aplicabilidad de los resultados, las carencias encontradas constituyeron un punto de partida para la elaboración de una estrategia de formación continua que respondiera a las necesidades formativas reales del profesorado.
\end{abstract}

Palabras clave: educación permanente, necesidades educacionales, personal académico docente, demanda de educación

Abstract: The proposal of a pedagogical model that meets the 21st century educational demands in the University of Artemisia, Cuba, demanded the identification of training needs of the teaching staff to implement the model, which justify this research and it becomes into its aim. A quantitative methodology was used to explore the needs of continuous training in the sample of teachers selected through a survey that included open and closed questions, as well as Likert-type scaling. The results indicate that, although not in all cases, the respondents were reflexive enough to recognize in themselves the educational deficiencies, these exist; On the other hand, limitations in the methodological work as an essential training activity were evident, and the teachers surveyed identified the lack of a continuous training offer by the University. As conclusions, the results obtained through the applied instrument represent only the first stage of the process of identifying training needs, these should be enriched with university policies for the elective period, the results reports of the methodological work of the previous period, the results of the annual evaluations of teachers and of the controls to classes realized to the teachers. In addition, like any process, it must be fed and fed back with certain regularity so that its cycle is permanently updated. Regarding the applicability of the results, the deficiencies found constitute a starting point for the elaboration of a continuous training strategy that responds to the real training needs of teachers.

Key words: lifelong education, educational needs, competency based teaching, educational objectives

1 Director del Centro de Estudios de Educación y Desarrollo de la Universidad de Artemisa, Artemisa, Cuba. Dirección electrónica: andresri1955@gmail.com Orcid https://orcid.org/0000-0002-2879-4469

2 Profesora del Centro de Estudios de Educación y Desarrollo de la Universidad de Artemisa, Artemisa, Cuba. Dirección electrónica: bertham@uart.edu.cu Orcid https://orcid.org/0000-0002-6750-8314

3 Profesor del Centro de Estudios de Educación y Desarrollo de la Universidad de Artemisa, Artemisa, Cuba. Dirección electrónica: yosdey@uart.edu.cu Orcid http://orcid.org/0000-0002-9769-0938

Artículo recibido: 16 de junio, 2020

Enviado a corrección: 18 de setiembre, 2020

Aprobado: 9 de noviembre, 2020 


\section{Introducción}

\subsection{La justificación de un diagnóstico de necesidades formativas del profesorado}

Una de las características esenciales de la sociedad del siglo XXI es que el conocimiento se convierte en uno de los principales valores de los sujetos, lo que conlleva un proceso de transformaciones que afecta la forma en que la ciudadanía se organiza, trabaja, se relaciona y aprende (Marcelo, 2001, p. 532). Estos cambios se reflejan en la escuela como institución encargada de formar a los ciudadanos y las ciudadanas:

Los cambios que se producen en el conocimiento, así como sus implicaciones en la vida productiva y los servicios, exigen al sistema educativo en todos sus niveles que se desarrollen en los estudiantes la capacidad de adaptarse a los rápidos cambios que experimenta la sociedad, preparación para cambiar el entorno en que se insertan, así como que sean creativos e innovadores en la aplicación de conocimientos para resolver problemas sociales y laborales. (Goel, 2019, p. 432)

En esta dirección resulta de particular importancia la formación en pregrado de profesionales, la cual tiene lugar en las universidades, pues la preparación que reciben las personas egresadas en el futuro en este período es crucial para su incorporación a la producción y los servicios, quienes deberán contar con una mentalidad innovadora para solucionar los problemas profesionales y sociales que enfrentarán en su vida laboral. En consonancia con la necesidad de la formación en pregrado de profesionales se ha desarrollado el proyecto de investigación Modelo pedagógico acorde con las demandas educativas del siglo XXI para la formación de profesionales en la Universidad de Artemisa. Uno de los resultados de este proyecto caracteriza el proceso de enseñanza-aprendizaje (PEA) en este nivel de enseñanza, para dar respuesta a las demandas educativas de la sociedad cubana del siglo XXI (Rodríguez et al., 2019). 
El éxito de la implementación práctica de este resultado de investigación depende de varios factores como la existencia de materiales docentes y medios de enseñanza-aprendizaje adecuados, y/o el perfeccionamiento de los programas de asignaturas y disciplinas. Sin embargo, como apuntan Yolcu y Kartal (2017, p. 918), el factor más importante a considerar para que el sistema educacional funcione con calidad es el profesorado, pues la preparación estudiantil, demandada por la sociedad del siglo XXI, solo será posible si quien enseña alcanza un desarrollo profesional que le posibilite dirigir dicha preparación. Este hecho es corroborado por Barber y Mourshed (2008, p. 302) cuando aseveran que "la calidad de un sistema educativo se basa en la calidad de sus docentes". En este empeño son esenciales las actividades de formación continua del profesorado en ejercicio, pues ellas posibilitan que la preparación del personal docente se ubique en congruencia con dichas exigencias sociales (Estrada, 2016, p. 5). La formación continua, como proceso de desarrollo profesional de docentes a lo largo de la vida, según Goel (2019, p. 433), "es necesitada para equiparlos con conocimientos y habilidades profesionales, así como con las actitudes necesarias para una enseñanza efectiva".

Las actividades formativas pueden ser muy variadas, pues en ellas se incluyen tanto aquellas programadas por la Institución de Educación Superior (IES) en que se desempeña la persona docente como otras que esta persona elige libremente para satisfacer sus necesidades. Las que programa la IES deben responder, según González y González (2007, p. 9) a determinadas exigencias para que se establezcan como estrategias de formación del profesorado: contribuir a la formación integral del profesorado, vincular la teoría y la práctica profesional, ser flexibles, contextualizadas y propiciar la reflexión del profesorado, además de asumir el compromiso con la calidad del desempeño profesional en ambientes de diálogo.

Se asume la definición de estrategia de formación docente dada por González y González, quienes la definen como "acciones educativas en el ejercicio de la profesión dirigidas a potenciar el desarrollo profesional del docente, es decir, a lograr su autodeterminación en el desempeño profesional” (González y González, 2007, p. 9). No obstante, se adecua a esta investigación. De esta manera, se define la estrategia de formación continua del profesorado para el ejercicio de las funciones con pregrado como el sistema de acciones educativas en el ejercicio de la profesión dirigido a potenciar el desempeño profesional docente, a partir de las potencialidades y necesidades, en relación con las demandas educativas del siglo XXI para los profesionales universitarios en formación. 
Según Valle (2012, p. 189), las estrategias en general constan de tres etapas fundamentales: diagnóstico, planeación-instrumentación y evaluación. Este estudio se enfoca en la primera etapa de la estrategia de formación continua del profesorado para la formación de profesionales, pues la identificación de necesidades formativas del profesorado es parte del diagnóstico a realizar.

El diseño de las estrategias de formación parte, inevitablemente, de las necesidades del profesorado y, por ello, su identificación es un factor primordial a tener en cuenta, pues como aseveran González y González (2007, p. 4): "nos permite establecer parámetros sobre las deficiencias con el fin de establecer mejores elementos correctores". La identificación de necesidades formativas permite conocer aquellos aspectos en que el profesorado presenta carencias que le pueden obstaculizar un desempeño profesional competente respecto a lo que se espera por la institución. Dichas necesidades formativas pueden variar de un docente a otro en relación con las exigencias sociales, y es su identificación lo que posibilita determinar, tanto aquellas que se localizan en determinado profesorado como las de carácter general, aunque estas últimas tienen un mayor valor si el fin es el diseño de una estrategia de formación continua en la IES.

La determinación de las necesidades formativas del profesorado es el punto de partida para la elaboración de los programas formativos que permitirán implementar una estrategia de formación continua que potencie, en los miembros del claustro, un desempeño profesional competente en la aplicación práctica del modelo pedagógico propuesto para la formación de profesionales en su fase de pregrado en la Universidad de Artemisa. Ello justifica que se formule como objetivo de esta investigación: identificar las necesidades formativas del profesorado de la Universidad de Artemisa en relación con el cumplimiento de las demandas educativas del siglo XXI para la formación de profesionales universitarios. Para darle cumplimiento, la indagación se desarrolló en el segundo semestre del año 2019.

\section{Referente teórico}

\subsection{Las demandas educativas de la sociedad del siglo XXI}

La educación contribuye notablemente al desarrollo integral de las personas, particularmente a la adquisición de conocimientos, habilidades y competencias necesarias para ser miembro de una sociedad. En este sentido, el sistema educativo está encargado de formar a las personas para convivir con los cambios derivados de las mejoras tecnológicas y de la globalización, y en ello, el profesorado desempeña uno de los más importantes roles, al 
ser una pieza clave para el trabajo interactivo, colaborativo y productivo con aprendices, programas de estudio, proceso de enseñanza-aprendizaje y colectivo de docentes (Solak y Çer, 2018, p. 42).

El profesorado requiere de una formación continua que le permita desempeñar cabalmente ese importante rol, pues, como señalan Sakkoulis, Asimaki y Vergidis (2018, p. 48), las actividades formativas "componen un continuo educacional que interactúa permanentemente con el ambiente socioeconómico, político y cultural en que se enmarca el sistema educativo en un determinado momento". De este modo, se justifica el diseño de una estrategia de formación continua del profesorado en función de prepararlos para cumplir cabalmente, mediante su práctica educativa, con las demandas educativas de la sociedad del siglo XXI.

Núñez, Solano, González-Pienda y Rosário (2006, p. 139) consideran que las principales demandas a las cuales debe dar respuesta el sistema educativo en el siglo XXI son básicamente dos: lograr que los educandos alcancen el desarrollo máximo de todas sus capacidades y prepararlos para enfrentar los retos que se derivan de la convivencia en la sociedad del conocimiento que caracteriza este siglo. De esta manera, estos autores conciben, además de la formación de profesionales competentes, la necesidad del desarrollo integral de sus personalidades, concepción que se comparte en esta investigación.

Horruitiner (2006, pp. 31-33) caracteriza el proceso de formación de pregrado en la Educación Superior de Cuba mediante la identificación de tres dimensiones esenciales (instructiva, desarrolladora y educativa), las cuales, integradas, expresan el tipo de profesional a formar para un desempeño competente en la sociedad. La dimensión instructiva responde a la necesidad de dotarlo con los conocimientos y las habilidades esenciales de su profesión, para desempeñarse en un determinado puesto de trabajo.

La dimensión desarrolladora se refiere a que, además de instruirse durante su formación de pregrado, el estudiante o la estudiante debe ponerse en contacto con el objeto de su profesión desde los primeros años de su carrera para que logre los nexos requeridos con los modos de actuación de esa profesión, con lo que se asegura las habilidades profesionales necesarias para un desempeño competente. La dimensión educativa abarca las dos primeras, y va más allá, pues se trata de que, mediante el contenido de las diferentes disciplinas, se establezca el vínculo con la profesión, la interacción estudiantil y con otras esferas de la vida universitaria, es decir, que el estudiantado tenga la posibilidad de adquirir no solo los 
conocimientos y habilidades requeridos por la profesión, sino también las actitudes, valores y sentimientos que demanda la sociedad cubana del siglo XXI.

De lo anterior se deduce que la meta de las universidades cubanas para la formación profesional, en la etapa de pregrado, es preparar a quienes se egresasan en el futuro para un desempeño competente, lo cual, como se puede apreciar, está en consonancia con los desafíos sociales que debe afrontar la formación de profesionales en la educación terciaria (Cuevas 2016, pp. 102-103). El logro de esta meta conduce al necesario cumplimiento de ciertas demandas educativas que abarcan tanto del tipo cognitivo-instrumentales como axiológicas.

Las demandas educativas de la sociedad cubana del siglo XXI han sido identificadas como parte de un resultado del proyecto investigativo Modelo Pedagógico acorde con las demandas del siglo XXI para la formación de profesionales en la Universidad de Artemisa (Rodríguez y Miqueli, 2019, p.116):

Axiológicas: desarrollo de la responsabilidad profesional, social y laboral, solidaridad, honestidad, dignidad, patriotismo, humanismo, honradez, justicia social, conciencia de la necesidad del cuidado y conservación del medio ambiente para el desarrollo sostenible y respeto a la diversidad.

Cognitivo-instrumentales: desarrollo del pensamiento crítico, independencia para la toma de decisiones, autonomía para el aprendizaje a lo largo de toda la vida, habilidades laborales e investigativas para la solución creativa de problemas profesionales, dominio de las Tecnologías de la Información y la Comunicación (TIC) como fuente y medio de aprendizaje, así como en función del desempeño laboral e investigativo, habilidades para el trabajo en equipos a partir de la cooperación y ayuda, dominio de lenguas extranjeras.

En la práctica educativa ambos tipos de demandas deben integrarse para propiciar el desarrollo integral de la personalidad del estudiantado, lo que requiere adquirir conocimientos y habilidades en estrecha armonía con la formación de sentimientos, motivaciones, valores y actitudes. Por lo tanto, en el proceso de formación de pregrado, como señalan Castellanos et al. (2007, p. 63), se requiere la unidad y equilibrio de lo cognitivo y lo afectivo-valorativo.

Por una parte, las demandas axiológicas contribuyen a la necesaria formación humanista de las futuras personas egresados del pregrado y no se logran automáticamente de un proceso de exposición de las personas a determinada información o experiencia. Cada una de estas 
demandas constituye una formación psicológica compleja, cuya apropiación solo es posible mediante un proceso relativamente largo, que requiere:

de la implicación activa de los sujetos hacia los aprendizajes que realizan, de la oportunidad de valorar de manera muy personal sus contenidos desde diversos puntos de vista y perspectivas (moral, social, ideológica), de analizar sus consecuencias y de reflexionar sobre cómo se vinculan dichos contenidos con sus propias conductas, con su mundo afectivo y con sus auténticas necesidades de interacción con el medio circundante. (Castellanos et al., 2007, p. 64)

Por otra parte, las demandas cognitivo-instrumentales constituyen el sustento del egreso de un profesional capaz de gestionar información y convertirla en conocimientos para solucionar problemas identificados en su realidad profesional y social, que es una condición para ser competente en la sociedad del siglo XXI, lo que requiere del estudiantado el desarrollo de un aprendizaje autorregulado (Zimmerman, 2008, p. 167). Este tipo de aprendizaje, según Bozpolat, (2016, p. 302), tiene sus fundamentos en la concepción estudiantil como parte activa del proceso de enseñanza-aprendizaje; es decir, que el estudiantado piensa, explora, pregunta, produce, toma decisiones y asume la responsabilidad y control de su aprendizaje, que incluye lo que aprende y cómo lo aprende.

Entre estas demandas se considera que el dominio de las TIC es fundamental, pues su empleo eficaz permite solucionar problemas profesionales complejos, exige la ejercitación constante en la toma de decisiones y del pensamiento crítico al adentrarse en el inmenso volumen de información al que, de modo fácil y rápido, permiten acceder (Rodríguez et al., 2019 , p. 7). Además, constituye una poderosa herramienta tanto para el aprendizaje autónomo (Hernández, 2014, p. 25) como para establecer colaboración con otros y desarrollar trabajo en equipos (Colorado-Aguilar y Edel-Navarro, 2015, p.3). También, un empleo óptimo de las TIC requiere de un dominio aceptable del inglés como lengua extranjera, a la vez que su empleo facilita el aprendizaje de esta lengua (Texidor Pellón, Reyes Miranda, Berry González y Cisnero Reyna, 2017, p. 4). Los efectos positivos de las TIC sobre el aprendizaje del estudiantado dependen de la preparación del profesorado para relacionarlas con el resto de las variables curriculares y de las características de los y las aprendices (Cabero, 2003, p. 27).

Solo si el personal docente adquiere la suficiente preparación logrará el cumplimiento de las demandas educativas con sus estudiantes mediante su práctica educativa, y una vía importante para ello la constituye la formación continua del profesorado. 


\subsection{La formación continua del profesorado}

González y González (2007, p. 8) identifican la formación continua del profesorado simplemente como formación docente, mientras que Quintero Tapia, Miranda Jaña y Rivera Rivera $(2018$, p. 6) prefieren denominarla formación permanente. No obstante, existe consenso entre estos autores en que se trata de un proceso de aprendizaje a lo largo de toda la vida del personal docente, que contribuye al desarrollo profesional de alta calidad, lo que incluye no solo la construcción de conocimientos y habilidades profesionales, sino también de actitudes, valores, vivencias y motivaciones que le permitan actuar profesionalmente de forma responsable y ética.

En este estudio se emplea el término formación continua del profesorado por estar más a tono con la terminología general utilizada por el Ministerio de Educación Superior (MES) de Cuba, que designa el término formación continua de profesionales para referirse al aprendizaje profesional que debe acompañar a lo largo de su vida a los graduados de diferentes carreras y perfiles universitarios (Ministerio de Educación Superior, MES, 2016, p. 4).

Más que la terminología empleada lo significativo es la meta que persigue la estrategia: elevar el desarrollo profesional del profesorado participante. De manera tal que se traduzca en su desempeño profesional competente, ético y comprometido. En tal sentido, Öztürk (2019, p. 25) se refiere a dos pilares que deben sustentar estas estrategias, el primero tiene en cuenta las características de las prácticas que conducen a un desarrollo profesional de alta calidad en el profesorado, y el segundo se refiere a que los cambios que se introducen en las prácticas educativas deben ser significativos para el profesorado.

Respecto al primer pilar existen dos tipos de características de los programas de formación continua. El primer tipo se refiere a características del contenido que se trata: debe estar contextualizado con la práctica educativa y centrado fundamentalmente en el aprendizaje estudiantil (Öztürk, 2019, p. 25). Según Borko, Jacobs y Koellner (2010, p. 550), una posible vía para cumplir con esta característica podría ser iniciar las actividades formativas con la presentación de problemas de la práctica educativa vinculados a la experiencia de personal docente participante, esto para valorar posibles soluciones a los problemas, potenciar la toma de decisiones sobre la mejor solución, así como generalizar a otras situaciones, en lugar de comenzar por el tratamiento de aspectos abstractos del contenido del programa formativo.

El segundo tipo de características está referido al proceso de implementación de los programas concebidos. Según Goel (2019, p. 433), "los profesores usualmente requieren un 
ambiente de confianza mutua, aprendizaje y apoyo para su desarrollo profesional, en que cada docente busque su excelencia y la de sus estudiantes". Ello demanda involucrar a al profesorado participante de las actividades formativas en un aprendizaje activo y concebir la construcción de una comunidad de aprendizaje profesional ético y responsable entre los presentes. Entre las vías para lograrlo se destacan el empleo del trabajo en equipos y del aprendizaje cooperativo entre docentes.

En relación con el segundo pilar, estrechamente vinculado con el primero, el profesorado debe sentir que los cambios que se desean introducir en el PEA, a partir del modelo pedagógico para mejorar la práctica educativa, son significativos para ellos. Como la atribución de significado es personal, debe asegurarse la convicción y el compromiso de los participantes con los cambios a implementar para que se involucren en ellos por libre voluntad. Para lograrlo es indispensable tener en cuenta lo que el profesorado piensa, lo que ya sabe y lo que necesita para sus prácticas educativas habituales (Bayar y Kösterelioğlu, 2014, pp. 327-328).

Resulta esencial, entonces, la identificación de necesidades formativas, y una vez conocidas estas, otorgarle al equipo docente participante el poder de decisión acerca de lo que debe hacerse. Se trata de explotar la inteligencia individual y colectiva del profesorado para solucionar los problemas educativos. En vez de ofrecerles orientaciones concretas de cómo hacerlo, estimular su iniciativa para la búsqueda de las soluciones, lo que resume Duffy (1994, p. 596) al plantear que, en vez de decirles qué hacer es mejor empoderarlos para que decidan por sí mismos lo que debe hacerse; es decir, es preferible invertir en las mentes docentes en lugar de hacerlo en un conjunto de orientaciones para que las sigan.

Estos pilares se integran mediante una formación continua del profesorado, que otorgue especial atención a las creencias y experiencias docentes acerca del proceso de enseñanzaaprendizaje como punto de partida para el logro del cambio deseado en sus prácticas educativas. Además, la implementación de los programas formativos debe estimular la crítica y la autorreflexión acerca de la práctica educativa, de manera que los participantes tomen el control de su propio aprendizaje y sean fuentes de información para la construcción de los conocimientos y habilidades profesionales necesarios para propiciar el cambio.

De ahí que, la formación continua del profesorado ha de ser concebida como un proceso educativo flexible y contextualizado (González y González, 2007, p. 10). Por ello, es importante, en el diseño de una estrategia de formación continua, concebir diferentes alternativas que se ajusten, tanto a las necesidades y potencialidades del profesorado en 
relación con las demandas educativas de la sociedad del siglo XXI como a las exigencias del contexto de actuación profesional.

Según estas propias autoras, son variadas las alternativas formativas que se pueden adoptar para llevar a cabo la estrategia de formación continua: conferencias-coloquios, mesas redondas, paneles, cursos, seminarios, entrenamientos, talleres, foros de discusión y jornadas científico-metodológicas, entre otras. Cada una de ellas, presentada en diferentes modalidades, presencial, semipresencial y a distancia (González y González, 2007, p. 10). La elección de las alternativas apropiadas dependerá, en gran medida, de los resultados de la identificación de necesidades formativas realizadas y del contexto en que se realicen.

\subsubsection{El trabajo metodológico como parte de la formación continua del profesorado}

La calidad del PEA que llevan a cabo el equipo docente con sus estudiantes depende, en gran medida, de la preparación previa que realice de la clase y dicho aseguramiento de la docencia se inserta en lo que, en Cuba, se denomina trabajo metodológico. Este tipo de trabajo es parte de la formación continua que realiza el profesorado, tanto de forma individual como de forma colectiva, mediante la participación de docentes en reuniones de asignatura, de disciplina, de carrera, de año académico, de departamentos docentes y de facultades, entre otros.

El Reglamento del Trabajo Docente y Metodológico (Resolución ministerial 2/2018) emitido por el MES, caracteriza al trabajo metodológico y traza los lineamientos fundamentales para desarrollarlo. Este se define como:

Labor que, apoyados en la Didáctica, realizan los sujetos que intervienen en el proceso docente educativo, con el propósito de alcanzar óptimos resultados en dicho proceso, jerarquizando la labor educativa desde la instrucción, para satisfacer plenamente los objetivos formulados en los planes de estudio. (MES, 2018, p. 5)

En este mismo documento (MES, 2018, p. 5) se identifican, como sus momentos principales, la planificación, la ejecución y el control. El adecuado desarrollo de estos requiere la participación activa de docentes, personal directivo y personal de apoyo, como vía de garantizar la calidad del proceso de formación, que se concreta en una apropiada integración de las clases con la actividad investigativa y laboral, las tareas de alto impacto social y las de carácter extracurricular que cumple el estudiantado.

Según el MES (2018, p. 5), el trabajo metodológico que se realiza de forma individual parte de la autopreparación dirigida e intencionada que realiza cada docente en los aspectos 
científico-técnico, filosófico, político-ideológico, didáctico y pedagógico necesarios para el desarrollo de su labor docente. En su forma colectiva, el trabajo metodológico tiene enfoque sistémico y se lleva a cabo para todos los tipos de curso y en cada uno de los niveles organizativos del proceso docente educativo.

Se identifican, como niveles organizativos del proceso docente educativo, de acuerdo con el MES (2018, pp. 6-20), los siguientes: Colectivo de carrera, Colectivo de disciplina, Colectivo de asignatura y Colectivo de año. Cada uno de ellos tiene funciones específicas que están en congruencia con su denominación. El colectivo de año constituye un nivel de dirección atípico en la estructura de las instituciones de Educación Superior, conducido por el profesor principal del año académico. Agrupa al profesorado que desarrolla las asignaturas del año, a docentes guías de cada grupo, a tutores y tutoras y a representantes de las organizaciones estudiantiles. Este colectivo de año académico tiene como propósito lograr el cumplimiento de los objetivos de formación del año académico, así como otros que se hayan determinado en respuesta a las características propias del grupo y del momento mediante el diseño e implementación de una estrategia educativa del año académico.

El trabajo metodológico se realiza también en diferentes niveles de dirección: institución de educación superior; facultad, instituto, colegio y centro universitario municipal (CUM); filial o unidad docente y departamento docente. Estos niveles, según el MES (2018, p. 21), son los encargados de atender y controlar esta labor en los colectivos de carrera, disciplina y asignatura, y año académico, según corresponda, y evaluar sus resultados.

Las formas fundamentales del trabajo metodológico son: Docente-metodológico y Científico-metodológico; ambas se integran, como sistema para el logro de los objetivos propuestos. De acuerdo con el MES (2018, pp. 22-24), el trabajo docente-metodológico es la actividad que se realiza con el fin de atender, en general, las principales prioridades y necesidades del proceso docente-educativo a partir de la preparación didáctica que posee el profesorado, así como de su experiencia acumulada. Por su parte, el trabajo científicometodológico se encamina a perfeccionar el proceso docente educativo a partir de investigaciones o mediante la utilización de los resultados de investigaciones realizadas, que contribuyan a la formación integral de los futuros profesionales.

Dada la importancia del trabajo metodológico para el logro de una formación profesional de alta calidad del profesorado se hace necesario identificar las necesidades formativas para concebir alternativas mediante las cuales estas necesidades puedan ser atendidas adecuadamente como parte de la estrategia de formación continua del profesorado. 


\subsection{Métodos para la identificación de necesidades formativas del profesorado}

Según Almarshad (2017, p. 175), entre los métodos para la identificación de necesidades formativas del profesorado se incluyen: entrevistas, informes de eficiencia, evaluación del desempeño y encuestas. Las entrevistas deben ser individuales para la detección de las necesidades formativas de cada docente en particular. Los informes de eficiencia son elaborados por los supervisores para destacar las carencias y potencialidades docentes en particular, en ellos se describe el estado de sus conocimientos, experiencia, actitud y comportamiento, por lo que proporcionan una clara información que posibilita determinar las necesidades formativas docentes.

La evaluación del desempeño es un método de identificación de deficiencias mediante la comparación del desempeño real con el esperado para identificar las necesidades formativas (Almarshad, 2017, p. 175). Tanto los informes de eficiencia como la evaluación del desempeño pueden tener como punto de partida la evaluación anual del profesorado que, por lo general, realizan las IES, anualmente, de cada docente Estos métodos (entrevista, informes de eficiencia y evaluación del desempeño) pueden ser muy efectivos cuando la estrategia formativa estará dirigida a un reducido número de docentes.

La encuesta es una vía de recolección de datos que refleja la actitud de una persona hacia cierto aspecto. Puede incluir frases y preguntas abiertas o cerradas. Constituye un importante método de identificación de necesidades formativas del profesorado al aventajar a los métodos descritos anteriormente en que puede involucrar a un elevado número de docentes y, a la vez, darles la oportunidad de expresar sus necesidades sin presión alguna, al tener libertad e independencia para hacerlo. (Almarshad, 2017, p. 175).

Fernández, Valdés y González (2008, p.79) reportan otras vías particulares para el diagnóstico de necesidades relacionadas con el trabajo metodológico: a) la revisión de las prioridades del Ministerio de Educación Superior para dicho período lectivo y b) los resultados del desarrollo y evaluación del trabajo metodológico del período anterior.

\section{Metodología}

\subsection{Enfoque}

La complejidad que implica el estudio de la formación continua del profesorado posibilitó asumir una complementariedad metodológica mediante un enfoque cuantitativo. Se empleó un diseño investigativo de triangulación concurrente para la recolección, análisis e 
interpretación de la información, y la investigación tuvo un alcance descriptivo y exploratorio, (Hernández Sampieri, Fernández Collado y Baptista Lucio, 2014, pp. 92, 534 y 557).

\subsection{Unidades de análisis}

La población la conformó el profesorado de la Universidad de Artemisa, de la provincia Artemisa, Cuba (250 docentes). Como único criterio de inclusión se empleó el hecho de ser docente de esta institución, ya sea a tiempo completo o tiempo parcial. Con la pretensión de incluir en la muestra al mayor número de docentes posible, se les solicitó a los jefes de Departamento Docente y a los directores de los Centros Municipales Universitarios (CUM) que convocaran a todo el profesorado universitario dispuesto a participar en el estudio, garantizando así el consentimiento informado de los participantes y la posibilidad de participación de todo el personal docente en la determinación de las necesidades de formación continua. De esta manera, se llevó a cabo un muestreo concurrente para métodos mixtos (Hernández Sampieri, Fernández Collado y Baptista Lucio, 2014, p. 568), pues la muestra se empleó simultáneamente para recopilar tanto información cuantitativa como cualitativa. Solo 29 docentes respondieron la encuesta, quienes conformaron la muestra de estudio.

Se incluyeron en la muestra representantes de todos los departamentos docentes y de siete de los 11 CUM que conforman la Universidad de Artemisa. De ellos, el 100\% son docentes en tiempo completo y el $79 \%$ sobrepasa los 10 años de experiencia en la docencia universitaria. A pesar de que fue relativamente bajo el porcentaje de personas encuestadas respecto al total del profesorado de la Universidad (11,6\%), estuvieron representados el $100 \%$ de los departamentos docentes y la mayoría de los CUM (63,6\%). Ello permite considerar las necesidades formativas identificadas en esta muestra representativa del profesorado en las diferentes áreas universitarias. El estudio se desarrolló en el segundo semestre de 2019.

\subsection{Técnicas de recolección}

Para determinar las necesidades formativas que pueden existir en el profesorado de la Universidad de Artemisa para implementar los principales resultados del proyecto de investigación Modelo pedagógico acorde con las demandas educativas del siglo XXI para la formación de profesionales, se elaboró un cuestionario en el que se combinaron preguntas de respuestas cerradas, de respuestas abiertas y el empleo de escalas tipo Likert.

Se exploraron los siguientes aspectos: conocimiento y metodología a emplear para el trabajo docente con las demandas educativas del siglo XXI (tanto cognitivo-instrumentales, como axiológicas); el empleo de la evaluación del aprendizaje y la tutoría de estudiantes como 
vías fundamentales para el desarrollo de la autorregulación del aprendizaje; el empleo de las TIC en el proceso de enseñanza-aprendizaje (PEA); la planificación, la organización, regulación y el control del trabajo metodológico mediante sus diferentes niveles organizativos, como vía de preparación para la docencia. Además, se indagó acerca de la periodicidad con que el profesorado participa en actividades formativas y los factores que obstaculizan su participación sistemática en ellas, con vistas a concebir una estrategia de formación continua que realmente responda a las necesidades e intereses del profesorado y al contexto en el que se desarrollan.

\subsection{Procesamiento de análisis}

En la validación de su contenido se siguió un procedimiento semejante al llevado a cabo por Almarshad (2017, p. 179): el cuestionario se entregó a diez expertos en el campo educativo, especialmente con vasta experiencia en la formación de docentes, para asegurar una correcta redacción, así como la correspondencia de los diferentes ítems con posibles necesidades formativas vinculadas a las demandas educativas del siglo XXI y, en particular, con los aspectos que se pretendía explorar, descritos en el párrafo anterior.

Se realizaron omisiones, adiciones y modificaciones hasta que el cuestionario contó con la validez de contenido de acuerdo con las valoraciones de los expertos. La consistencia interna o confiabilidad del instrumento se determinó mediante el coeficiente de correlación multidimensional rpj. Este coeficiente toma valores entre 0 y 1 , y el instrumento tiene consistencia interna si el valor del coeficiente es mayor o igual que 0,576. El valor de rpj se calcula fácilmente empleando el software "Cohaerentia" (Pérez Jacinto, 2017, p. 40) mediante un pilotaje del instrumento. El cálculo del rpj arrojó un valor de 0,625 , por lo que resultó confiable. Se consideran limitaciones del estudio realizado el hecho de que el tamaño de la muestra que se logró fue relativamente pequeño y, por otra parte, la magnitud del coeficiente rpj está cercano al límite de aceptación de la confiabilidad.

El cuestionario quedó conformado por 10 preguntas contentivas de un total de 63 incisos. En relación con las demandas educativas del siglo $\mathrm{XXI}$, se indagó con preguntas cerradas si el profesorado dominaba o no su significado, así como si les ofrecía dificultad o no el trabajarlas con el estudiantado. Las respuestas se procesaron mediante conteo de frecuencias, lo que posibilitó construir una tabla de frecuencias absolutas para las demandas cognitivoinstrumentales y otra para las axiológicas.

Con el ánimo de verificar si las personas encuestadas eran honestas en sus respuestas, se les cuestionó, mediante una pregunta abierta, acerca de los métodos que más comúnmente 
utilizaban en su práctica educativa para el trabajo en clase con las demandas. Las respuestas fueron procesadas mediante análisis de contenido (Kabadayi, 2016, p. 7; Ulla, 2018, p. 70), lo que implicó leerlas varias veces para entenderlas y asignarlas a diferentes categorías, que posibilitaron resumirlas como parte de los resultados encontrados.

Para explorar la frecuencia con que las personas encuestadas hacían uso de la evaluación del aprendizaje y la tutoría como vías de desarrollo del aprendizaje autorregulado en sus estudiantes, se les solicitó evaluar con una escala tipo Likert (5. Siempre, 4. Con frecuencia, 3. Algunas veces, 2. Pocas veces y 1. Nunca) los siguientes aspectos relacionados con estas vías: 1. diseño de instrumentos evaluativos que exigen el empleo del pensamiento crítico y la toma de decisiones; 2. planificación de evaluaciones no convencionales que fomentan la autorregulación del aprendizaje; 3. práctica de la autoevaluación y la coevaluación; 4. retroalimentación de los resultados evaluativos y 5 . tutoría de trabajos investigativos.

Los resultados se procesaron como medianas de los valores asignados por las personas encuestadas, a partir de la escala utilizada. Las medianas se calcularon con el software Cohaerentia, versión 1.0 (Pérez Jacinto, 2017, p. 40). Mediante la aplicación Microsoft Office Excel se construyó un gráfico en que se comparan las medianas de los aspectos evaluados. Este tipo de procesamiento de los datos se empleó para cada uno de los aspectos que fueron evaluados mediante la escala Likert descrita.

El empleo de las TIC fue explorado de forma semejante al desarrollo del aprendizaje autorregulado. Utilizando la misma escala Likert se indagó acerca de la frecuencia con que el profesorado emprendía las siguientes acciones relacionadas con el empleo de las TIC en sus clases: 1. como medio de enseñanza en la docencia presencial, 2. como contenido de la docencia, 3. utilizar entornos de aprendizaje de forma independiente, 4. tutorar estudiantes, 5. ampliar la comunicación docente-estudiante y entre estudiantes dentro y fuera del espacio áulico, 6. trabajar en equipos; Incrementar la participación estudiantil, 7. incrementar la participación estudiantil, 8. potenciar el liderazgo entre estudiantes, 9. en la solución de problemas profesionales y 10. para la evaluación y autoevaluación del aprendizaje.

La exploración del funcionamiento del trabajo metodológico se realizó mediante la evaluación del comportamiento de la participación del encuestado o de la encuestada en su planificación (aporte de criterios para la planificación del trabajo metodológico), ejecución (como participante y como ponente en actividades metodológicas), control y evaluación de las actividades metodológicas. Cada uno de estos aspectos fue evaluado para diferentes niveles: 
departamento docente, colectivo de carrera, colectivo de disciplina, colectivo de asignatura y colectivo de año. Se exploró, además, el grado en que el personal directivo de cada nivel tenía en cuenta las necesidades de quien se encuestó para el óptimo desarrollo de sus clases y la interrelación entre la realización del trabajo metodológico, el Modelo del Profesional a formar según la carrera y las demandas educativas de la sociedad del siglo XXI. Para la valoración se utilizó la escala Likert descrita anteriormente.

La periodicidad con que el profesorado participa en actividades formativas y los factores que obstaculizan su participación sistemática fueron explorados mediante preguntas cerradas, semicerradas y abiertas, que incluyeron, además, su disposición a participar y sugerencias para organizarlas atendiendo a sus intereses. Las respuestas cerradas se procesaron por conteo de frecuencias y las abiertas por análisis de contenido.

\section{Resultados y análisis}

\subsection{Demandas cognitivo-instrumentales}

El total de las personas encuestadas respondieron este aspecto de la encuesta, en que se les solicitó marcar aquellas demandas cognitivo-instrumentales cuya significación conocían plenamente y de ellas, aquellas que les costaban más o no sabían cómo potenciar en clases, así una misma demanda podía ser marcada en una de las opciones o en las dos. Los resultados obtenidos se resumen en la Tabla 1.

Tabla 1.

Frecuencias de respuestas de participantes sobre conocimiento y trabajo docente con demandas cognitivo-instrumentales, Universidad de Artemisa, Cuba, 2019

\begin{tabular}{|c|c|c|c|c|}
\hline \multirow[t]{3}{*}{ No. } & \multirow[t]{3}{*}{ Demandas } & \multicolumn{3}{|c|}{$\begin{array}{l}\text { Frecuencia de respuestas } \\
\qquad(n=29)\end{array}$} \\
\hline & & \multicolumn{2}{|c|}{ Conocimiento } & \multirow{2}{*}{$\begin{array}{c}\text { Carencias } \\
\text { Reconocen carencias para } \\
\text { potenciarlas en clases }\end{array}$} \\
\hline & & $\begin{array}{l}\text { Conocen su } \\
\text { significado }\end{array}$ & $\begin{array}{l}\text { Desconocen } \\
\text { su significado }\end{array}$ & \\
\hline 1 & $\begin{array}{l}\text { Aprendizaje } \\
\text { autorregulado }\end{array}$ & 26 & 3 & 6 \\
\hline 2 & Pensamiento crítico & 26 & 3 & 5 \\
\hline 3 & $\begin{array}{l}\text { Independencia para } \\
\text { la toma de } \\
\text { decisiones }\end{array}$ & 28 & 1 & 5 \\
\hline 4 & Trabajo en equipos & 29 & 0 & 0 \\
\hline 5 & Empleo de las TIC & 29 & 0 & 3 \\
\hline 6 & $\begin{array}{ll}\text { Solución } & \text { de } \\
\text { problemas } & \\
\text { profesionales } & \end{array}$ & 28 & 1 & 3 \\
\hline
\end{tabular}




\begin{tabular}{|l|l|l|l|l|}
\hline 7 & $\begin{array}{l}\text { Dominio de lenguas } \\
\text { extranjeras }\end{array}$ & 19 & 10 & 19 \\
\hline
\end{tabular}

Fuente: Elaboración propia a partir de los resultados de la encuesta

Como se aprecia, la inmensa mayoría (o la totalidad, en el caso de las demandas 4 y 5 ) manifestaron conocer a plenitud el significado de estas demandas, excepto en la demanda relacionada con el dominio de lenguas extranjeras en que el 34,5\% (10) expresó desconocimiento. En cuanto a los que reconocieron carencias en sí mismos para potenciar dichas demandas, mediante sus clases, se incluyeron por demanda todos los que marcaron desconocer el significado de la demanda y algunos que, a pesar de manifestar conocimiento de la demanda, declararon que tenían carencias para potenciarla en clases con sus estudiantes. Como se puede observar la frecuencia de este indicador es realmente baja (excepto en la demanda 7).

\subsection{Demandas axiológicas}

Todas las personas encuestadas respondieron este aspecto en que se solicitó a los participantes marcar aquellas demandas axiológicas cuya significación conocían plenamente y, de ellas, aquellas que les costaba más o no sabía cómo potenciar en sus estudiantes, así una misma demanda podía ser marcada en una de las opciones o en las dos. Los resultados se muestran en la Tabla 2.

Tabla 2.

Frecuencias de respuestas de participantes sobre conocimiento y trabajo docente con demandas axiológicas, Universidad de Artemisa, Cuba, 2019

\begin{tabular}{|l|l|c|c|c|}
\hline \multirow{2}{*}{ No. } & \multicolumn{1}{|c|}{ Demandas } & \multicolumn{3}{c|}{$\begin{array}{c}\text { Frecuencia de respuestas } \\
(\mathrm{n}=29)\end{array}$} \\
\cline { 3 - 5 } & & \multicolumn{2}{|c|}{ Conocimiento } & \multicolumn{1}{c|}{ Carencia } \\
\cline { 3 - 5 } & & $\begin{array}{l}\text { Conocen el } \\
\text { significado }\end{array}$ & $\begin{array}{l}\text { Desconocen el } \\
\text { significado }\end{array}$ & $\begin{array}{c}\text { Reconocen carencias } \\
\text { para potenciarlas en } \\
\text { clases }\end{array}$ \\
\hline $\mathbf{1}$ & $\begin{array}{l}\text { Responsabilidad profesional, } \\
\text { social y laboral }\end{array}$ & 29 & 0 & 1 \\
\hline $\mathbf{2}$ & Solidaridad & 29 & 0 & 0 \\
\hline $\mathbf{3}$ & Honestidad & 29 & 0 & 0 \\
\hline $\mathbf{4}$ & Honradez & 29 & 0 & 0 \\
\hline $\mathbf{5}$ & Dignidad & 29 & 0 & 0 \\
\hline $\mathbf{6}$ & Humanismo & 29 & 0 & 0 \\
\hline $\mathbf{7}$ & Patriotismo & & 0 & 1 \\
\hline $\mathbf{8}$ & $\begin{array}{l}\text { Conciencia del cuidado del medio } \\
\text { ambiente para el desarrollo } \\
\text { sostenible }\end{array}$ & 29 & 0 & 0 \\
\hline
\end{tabular}

Fuente: Elaboración propia a partir de resultados de encuesta 
Llama la atención que todas las personas encuestadas manifestaran pleno dominio del significado de estas demandas y que un solo caso haya reconocido carencias para potenciar su desarrollo mediante las clases en dos de las demandas, pues se trata en cada caso de formaciones psicológicas complejas.

\subsection{Autorregulación del aprendizaje}

Esta se trata de una de las demandas cognitivo-instrumentales que resulta central respecto al resto y por ello se profundiza en dos vías que resultan esenciales para lograr su desarrollo en el estudiantado: la evaluación del aprendizaje y la tutoría a estudiantes. Para determinar las necesidades formativas en relación con estas vías, los participantes evaluaron la frecuencia con que empleaban cinco estrategias diferentes para potenciar el desarrollo del aprendizaje autorregulado en sus estudiantes: diseño de instrumentos evaluativos; planificación de evaluaciones que fomentan la autorregulación del aprendizaje; práctica de la autoevaluación y la coevaluación; retroalimentación de los resultados evaluativos y tutoría de trabajos investigativos. Las personas encuestadas marcaron la categoría que mejor reflejaba la frecuencia con que empleaban cada estrategia en una escala (5. Siempre; 4. Con frecuencia; 3. Algunas veces; 2. Pocas veces; 1. Nunca). Se determinaron las medianas de las respuestas para cada estrategia y se muestran los resultados en la Tabla 3.

Tabla 3.

Valores de medianas que indican la frecuencia con que se emplean estrategias para potenciar aprendizaje autorregulado, Universidad de Artemisa, Cuba, 2019

\begin{tabular}{|l|c|}
\hline \multicolumn{1}{|c|}{ Estrategias } & Valores de medianas \\
\hline 1. Diseño de instrumentos evaluativos & 4,15 \\
\hline $\begin{array}{l}\text { 2. Planificación de evaluaciones que fomentan la } \\
\text { autorregulación del aprendizaje }\end{array}$ & 4,68 \\
\hline $\begin{array}{l}\text { 3. Práctica de la autoevaluación y la coevaluación } \\
\text { 4. Retroalimentación de los resultados } \\
\text { evaluativos }\end{array}$ & 4,72 \\
\hline 5. Tutoría de trabajos investigativos & 4,69 \\
\hline
\end{tabular}

Fuente: Elaboración propia a partir de resultados de encuesta

Se aprecia que las cinco estrategias se emplean en un intervalo de con frecuencia y siempre (mediana entre 3,94 y 4,72). Dos de las estrategias se acercan más a la categoría 
con frecuencia (más próximas al valor 4 que al 5 de la mediana): Diseño de instrumentos evaluativos (1), con mediana 4,15 y Tutoría de trabajos investigativos (5), con mediana 3,94, que resultó la más baja. Las otras tres (estrategias 2,3 y 4) están más cercanas a la categoría de siempre (más próximas al valor 5 que al 4 de la mediana). De esta manera, se reconocen algunas carencias, sobre todo en la elaboración de los instrumentos evaluativos, y, en menor medida, en la tutoría de trabajos investigativos. Por lo tanto, en general, constituyen estrategias de enseñanza-aprendizaje habituales las siguientes: la planificación de evaluaciones que fomentan la autorregulación del aprendizaje, la práctica de la autoevaluación y la coevaluación, así como la retroalimentación de los resultados evaluativos.

\subsection{Empleo de las Tecnologías de la Información y la Comunicación (TIC) en la docencia}

Es otra demanda cognitivo-instrumental de vital importancia, pues permea no solo el resto de estas, sino que tiene también marcada influencia sobre el desarrollo estudiantil de muchas de las demandas axiológicas. Para la determinación de las necesidades formativas del profesorado, en esta temática, los participantes evaluaron la frecuencia con que empleaban cada una de diez acciones relacionadas con el empleo de las TIC en la docencia, en la encuesta: emplearlas como medio de enseñanza en la docencia presencial; incluirlas como contenido de la docencia; utilizar de forma independiente entornos de aprendizaje; asesorar estudiantes; ampliar comunicación docente-estudiante y entre estudiantes dentro y fuera del espacio áulico; trabajar en equipos; incrementar la participación estudiantil; potenciar el liderazgo entre el estudiantado; solucionar problemas profesionales; evaluar y autoevaluar el aprendizaje. En la Tabla 4 se muestran los resultados obtenidos.

Tabla 4.

Valores de medianas que indican la frecuencia de utilización de diferentes acciones relacionadas con el empleo de las TIC en la docencia, Universidad de Artemisa, Cuba, 2019

\begin{tabular}{|l|c|}
\hline \multicolumn{1}{|c|}{ Acciones } & Valores de mediana \\
\hline $\begin{array}{l}\text { 1. Emplearlas como medio de enseñanza en la docencia } \\
\text { presencial }\end{array}$ & 4,00 \\
\hline 2. Incluirlas como contenido de la docencia & 4,16 \\
\hline 3. Utilizar de forma independiente entornos de aprendizaje & 3,90 \\
\hline 4. Asesorar estudiantes & 4,74 \\
\hline $\begin{array}{l}\text { 5. Ampliar comunicación docente-estudiante y entre } \\
\text { estudiantes dentro y fuera del espacio áulico }\end{array}$ & 4,18 \\
\hline 6. Trabajar en equipos & 4,72 \\
\hline 7. Incrementar la participación estudiantil & 4,74 \\
\hline 8. Potenciar el liderazgo entre el estudiantado & 4,64 \\
\hline 9. Solucionar problemas profesionales & 4,18 \\
\hline 10. Evaluar y autoevaluar el aprendizaje & 4,04 \\
\hline
\end{tabular}


Fuente: Elaboración propia a partir de resultados de encuesta

Para la evaluación de la frecuencia de empleo de cada acción, así como para el procesamiento de las respuestas se empleó la misma escala y el mismo procedimiento descritos en el epígrafe 4.3. Se aprecia que la acción que se emplea menos es la utilización de entornos de aprendizaje para el trabajo independiente del estudiantado (acción 3), cuya mediana $(3,9)$ se encuentra entre las categorías de algunas veces (3) y con frecuencia (4), lo que puede estar relacionado con carencias en la preparación individual de las personas encuestadas para emprender esta acción.

El resto de las acciones muestran medianas que se sitúan entre la categoría de con frecuencia (4) y siempre (5). De ellas, cuatro acciones presentan medianas superiores a 4,5 (4.asesorar estudiantes, 6. trabajar en equipos, 7. incrementar la participación estudiantil y 8. potenciar el liderazgo entre estudiantes), por lo que están más cercanas a la categoría de siempre, lo que conduce a pensar que estas personas encuestadas mostrarían una explotación máxima de las potencialidades de sus estudiantes en materia de la tecnología de que disponen, regularidad que no se aprecia en la mayoría de las aulas de la Universidad de Artemisa, en que predomina una docencia tradicional. El resto de las acciones están más cercanas a la categoría de empleo con frecuencia.

Seguido de estas demandas en el cuestionario se insertó una pregunta dirigida esencialmente a contrastar los criterios emitidos por quienes se encuestaron en las preguntas anteriores, al solicitarles que mencionaran metodologías empleadas en sus clases para potenciar el desarrollo de las demandas educativas de la sociedad cubana del siglo XXI.

El $41,4 \%$ de las personas encuestadas no mencionaron metodología alguna y el resto lo hizo de forma tan general, que no transmiten claramente el trabajo que desarrollan en su docencia: metodologías novedosas con empleo de las TIC, método práctico-consciente, métodos y procedimientos productivos, clases prácticas, evaluación formativa, formación personalizada y trabajo independiente.

\subsection{El trabajo metodológico como vía de preparación para la docencia}

El trabajo metodológico integra el proceso de enseñanza-aprendizaje de las diferentes asignaturas con las exigencias de la formación del estudiantado de acuerdo con el modelo del profesional de la carrera. De ahí que debe diseñarse este trabajo metodológico a partir de los objetivos propuestos y relacionarse con las demandas sociales. Este incluye diferentes etapas 
como la planificación, organización, regulación y control, así como diferentes niveles organizativos para hacerlo viable. Resulta esencial que el profesorado participe activa y sistemáticamente en las diferentes etapas y niveles.

En sus respuestas a la encuesta, los participantes evaluaron la frecuencia con que ocurren cuatro acciones diferentes con las actividades metodológicas: 1. aportar criterios para su planificación; 2. participar; 3. impartir actividades; 4. controlar y evaluar. Cada acción, a su vez, se evaluó en cinco niveles organizativos: departamento docente, colectivo de carrera, colectivo de disciplina, colectivo de asignatura y colectivo de año, en ese mismo orden.

Para la evaluación de cada acción y para el procesamiento de las respuestas se empleó la misma escala y el mismo procedimiento descritos en los epígrafes 4.3 y 4.4 . Los resultados se muestran en la Tabla 5.

Es una regularidad que el colectivo de carrera alcance los mayores valores de mediana en las diferentes etapas del trabajo metodológico, por lo que se puede plantear, a partir de los criterios encuestados, que este nivel de organización es el que mejor funciona en la Universidad de Artemisa.

Tabla 5.

Valores de medianas que indican la frecuencia con que ocurren las acciones para diferentes niveles del trabajo metodológico, Universidad de Artemisa, Cuba, 2019

\begin{tabular}{|l|l|c|}
\hline \multicolumn{1}{|c|}{ Nivel } & \multicolumn{1}{|c|}{ Acciones } & Medianas \\
\hline \multirow{5}{*}{ Departamento docente } & 1. Aportar criterios para su planificación & 4,17 \\
\cline { 2 - 3 } & 2. Participar & 4,66 \\
\cline { 2 - 3 } & 3. Impartir actividades; & 4,11 \\
\cline { 2 - 3 } & 4. Controlar y evaluar & 4,07 \\
\hline \multirow{5}{*}{ Colectivo de carrera } & 1. Aportar criterios para su planificación & 3,98 \\
\cline { 2 - 3 } & 2. Participar & 4,66 \\
\cline { 2 - 3 } & 3. Impartir actividades; & 4,69 \\
\cline { 2 - 3 } & 4. Controlar y evaluar & 4,18 \\
\hline \multirow{5}{*}{ Colectivo de disciplina } & 1. Aportar criterios para su planificación & 4,18 \\
\cline { 2 - 3 } & 2. Participar & 4,09 \\
\cline { 2 - 3 } & 3. Impartir actividades; & 2,90 \\
\cline { 2 - 3 } & 4. Controlar y evaluar & 2,98 \\
\hline \multirow{5}{*}{ Colectivo de año } & 1. Aportar criterios para su planificación & 2,86 \\
\cline { 2 - 3 } & 2. Participar & 2,70 \\
\cline { 2 - 3 } & 3. Impartir actividades; & 2,17 \\
\cline { 2 - 3 } & 4. Controlar y evaluar & 3,97 \\
\hline & 1. Aportar criterios para su planificación & 4,64 \\
\cline { 2 - 3 } & 2. Participar & 4,08 \\
\cline { 2 - 3 } & 3. Impartir actividades; & 4,06 \\
\cline { 2 - 3 } & 4. Controlar y evaluar & 4,08 \\
\hline
\end{tabular}

Fuente: Elaboración propia a partir de resultados de encuesta

Como se aprecia en la Tabla 5, la acción de más bajos valores de mediana es la tercera, referida a la frecuencia con que la población encuestada imparte actividades metodológicas 
en los diferentes niveles organizativos. Llama la atención que los más bajos valores de la mediana corresponden a la realización de actividades metodológicas en el colectivo de asignatura y en el colectivo de año.

Además, las personas encuestadas evaluaron la frecuencia de ocurrencia de otras dos acciones para todos los niveles en general: 5 . ¿Tienen en cuenta los directivos de estos niveles tus necesidades para el óptimo desarrollo del PEA? y 6 . ¿Se establece una relación armónica para la realización del trabajo metodológico entre el Modelo del Profesional y las exigencias concretas de la sociedad actual? Los valores de mediana para ambas acciones resultaron ser 3,92, lo que indica, por una parte, la existencia de limitaciones para considerar las necesidades individuales docentes por parte del personal directivo y, por otra, no se logra una total armonía entre lo que se aspira a alcanzar con el modelo del profesional y las exigencias concretas de la sociedad actual.

\subsection{Participación en actividades de formación continua}

Respecto a la frecuencia de participación en actividades de formación continua, se observa el predominio en la muestra de una baja periodicidad de participación toda vez que 17 de las personas encuestadas refieren no participar en lo absoluto (58,6\%), ocho participan una vez por semestre $(27,6 \%)$ y cuatro una vez por año $(13,8 \%)$.

Los profesores y las profesoras destacan, entre los factores que obstaculizan su participación, la falta de oferta por parte de la UA, la incompatibilidad con los horarios de clase, la lejanía de la sede central y los problemas con el servicio sistemático del transporte obrero.

El 100\% de la muestra considera necesario contar con una oferta de formación continua del profesorado de la UA en la temática de las demandas educativas de la sociedad cubana del siglo XXI. También el $100 \%$ de la población encuestada muestra disposición a involucrarse en dichas actividades. Entre las sugerencias que aportan las personas encuestadas para llevar a cabo las actividades de formación continua se encuentran: Organizar una actividad central en la Universidad de forma bimensual, trimestral o semestral, concentrada en varios días, que agrupe todas las carreras universitarias, con un recorrido de transporte que posibilite la participación de todo el personal docente, diplomados y cursos cortos de hasta tres meses de duración con sesiones semanales, una sesión mensual, una sesión quincenal y una sesión semanal.

\section{Discusión y Conclusiones}




\subsection{Discusión}

El hecho de que la mayoría de las personas encuestadas sean docentes de larga experiencia en la Educación Superior puede tomarse como señal de que las carencias formativas que se manifiestan en ellos también estarán presentes en los de menor experiencia, considerando que el tiempo de trabajo en la docencia contribuye apreciablemente en la formación continua del profesorado, como señala Öztürk (2019, p. 26).

Una debilidad de la selección de la muestra fue que no se incluyeron, entre las personas encuestadas, docentes en tiempo parcial (PTP). Ello es una evidencia de que estos tienen una limitada participación en otras actividades de la Universidad, distintas a su docencia directa, cuestión bastante generalizada en las universidades estatales de otros países, como reportan en su investigación López, García, Pérez, Montero y Rojas (2016, p. 25). Aunque el cuerpo docente a tiempo parcial no están representados en este estudio, habrá que considerar su inclusión en la estrategia de formación continua que se diseñe, pues resulta importante que este estrato del profesorado eleve su calidad como docente universitario, ya que son una fuente de transmisión de experiencia profesional que no se debe menospreciar en el proceso formativo de una carrera determinada.

En relación con el conocimiento y trabajo docente con las demandas cognitivoinstrumentales sería conveniente aclarar que las relativamente elevadas frecuencias en la demanda 7 pueden deberse a confusiones de los términos conocimiento de su significado y conocimiento de una lengua extranjera, pues se puede conocer lo que significa su dominio como parte de la formación estudiantil (que es lo que se pretendía que respondieran), sin necesidad de dominar la lengua como tal. Las bajas frecuencias presentadas en el reconocimiento para potenciar estas demandas en clases pueden deberse a un dominio no pleno de lo que significa el cumplimiento en la práctica educativa de estas, dada la complejidad y el alto grado de preparación que requiere el desarrollo de cada una de ellas mediante el PEA de una asignatura en particular.

Se considera que la mayoría del profesorado encuestado no valoró, en toda su magnitud, lo que significa el potenciar las demandas axiológicas mediante el PEA de sus respectivas asignaturas, pues se trata de formaciones psicológicas complejas que requieren de una elevada preparación del profesorado para insertarlas de forma armónica como parte del contenido de su asignatura, y demandan de un trabajo interdisciplinar y de un largo período de tiempo para que se logren resultados en el estudiantes (Castellanos et al., 2007, p. 64). 
Las frecuencias con que se emplean, por el profesorado, diferentes estrategias para el desarrollo del aprendizaje autorregulado indican voluntad en los participantes por contribuir al logro del aprendizaje autorregulado tan necesario como parte de la formación de profesionales en la sociedad del siglo XXI.

Aunque el emprendimiento de diferentes acciones en el empleo de TIC en la docencia marcha satisfactoriamente según los resultados de la encuesta, se considera que, independientemente de estos resultados, la temática del empleo de las TIC es obligatoria en la formación continua, puesto que ella evoluciona a gran velocidad y cada día aparecen diversas novedades con aplicación al sistema educativo en general, lo que exige una actualización permanente del profesorado en la búsqueda de un desempeño profesional de alta calidad. Es común que en muchas universidades se incluyan frecuentemente las TIC entre las temáticas de formación continua del profesorado, como reportan en su investigación Montes y Suárez (2016, p. 11).

El hecho de que el profesorado encuestado no haya ofrecido respuestas convincentes en relación con la metodología empleada para el trabajo docente con las demandas educativas conduce a pensar que, contrario a lo que expresaron en las preguntas precedentes, no existe una sistematicidad intencionada para el trabajo con dichas demandas en el aula. De esta forma, se refleja otra necesidad de formación continua: la población encuestada denotó que no realizan sistemáticamente una reflexión crítica sobre la práctica educativa que llevan a cabo, y como señalan González y González (2007, p. 3) esta actividad junto a la participación activa y el compromiso con la tarea educativa constituyen elementos esenciales en la formación continua del profesorado.

A la luz de estas evidencias, las personas encuestadas en la Universidad de Artemisa necesitan actividades formativas para la adopción de tendencias modernas de enseñanzaaprendizaje en un siglo XXI caracterizado por sus rápidos cambios y los grandes avances tecnológicos. Estos resultados son semejantes a los encontrados por Almarshad (2017, p. 184).

Los resultados muestran carencias en las fases de planificación, organización y ejecución del trabajo metodológico, pues, como se ha mencionado en párrafos anteriores, al caracterizar la muestra la mayoría de las personas encuestadas tienen suficiente experiencia en la Educación Superior como para impartir determinadas actividades metodológicas en los diferentes niveles organizativos, lo que entra en contradicción con lo que se manifiesta en la encuesta. 
El bajo valor de mediana en la frecuencia con que se imparten actividades metodológicas en el colectivo de asignatura puede tener dos posibles causas: en ocasiones existe un solo docente impartiendo la asignatura, en cuyo caso no tiene sentido la realización de actividades metodológicas, o que no funciona debidamente este nivel organizativo cuando existe más de un profesor. La baja mediana para el colectivo de año es una evidencia de las carencias que presenta, en la Universidad de Artemisa, este importante nivel de organización, que desempeña un rol central en el funcionamiento del eslabón de base. No se justifica que sean pocas las veces que el profesorado se involucre en la impartición de actividades metodológicas a este nivel.

Otro aspecto a destacar en estos resultados es el hecho de que la acción cinco no llegue a alcanzar la categoría con frecuencia, cuando debía ser que siempre se tenga en cuenta, por el personal directivo de los diferentes niveles organizativos, las necesidades individuales del profesorado para el óptimo desarrollo del proceso de enseñanza aprendizaje. En este mismo sentido, tampoco debía esperarse una categoría inferior a la de siempre para la acción seis, relacionada con el establecimiento, en la realización del trabajo metodológico, de un vínculo armónico entre el Modelo del Profesional y las exigencias concretas de la sociedad del siglo XXI.

Estos resultados están alejados de los esperados, pues como han demostrado en su investigación Fernández, Valdés y González (2008, p. 93), un sistema de acciones metodológicas bien desarrolladas con docentes tiene una influencia decisiva en el desempeño profesional y en el desarrollo motivacional y humano de los implicados, así como en la formación de un profesorado reflexivo, que son metas importantes a lograr como parte de la formación continua.

Las evidencias constatadas en relación con la participación del profesorado en actividades de formación continua conducen a afirmar que la UA no cuenta en este momento con una oferta de formación continua en que se puedan involucrar todos sus docentes, lo que se considera una carencia esencial si se quiere garantizar una formación de profesionales que responda a las demandas de la sociedad del siglo XXI. Las ofertas de formación continua que brinda la Universidad deben ser dinámicas, pues, como indican Al-Majed, Al-Kathiri, Al-Ajmi y Al-Hamlan (2017), se debe evitar el asumir cuáles son las necesidades formativas. En su lugar, se hace necesario aplicar encuestas anuales al profesorado para reajustar las ofertas formativas en función de las tendencias en las necesidades que se identifiquen. 


\subsection{Conclusiones}

Las características del siglo XXI producen ineludiblemente cambios en la Educación Superior, específicamente en la Universidad de Artemisa. Este siglo ha estado signado por lo que se ha dado en llamar "sociedad del conocimiento". En esta situación, el proyecto de investigación Modelo pedagógico acorde con las demandas educativas del siglo XXI para la formación de profesionales en la Universidad de Artemisa ha identificado demandas educativas cognitivo-instrumentales y axiológicas que se hace necesario desarrollar como meta del proceso de formación de pregrado en el estudiantado. Entre los diversos factores que afectan el cumplimiento de esta meta, el más importante a considerar es el profesorado, pues la formación del estudiantado, demandada por la sociedad del siglo XXI, solo será alcanzable si el profesorado logra un desarrollo profesional de alta calidad, que le posibilite dirigir el proceso formativo del estudiantado en la dirección esperada. Para ello, el profesorado universitario debe acomodarse a los cambios sociales a partir de un cambio en las concepciones básicas de enseñanza-aprendizaje. Asimismo, dicho profesorado necesita reaprender para desarrollar estrategias pedagógicas que favorezcan el desarrollo de las demandas educativas de este siglo.

El proceso de reaprender del profesorado universitario va acompañado, inevitablemente, de su formación continua, entendida esta como el aprendizaje profesional que debe acompañar a lo largo de su vida al profesorado de la Educación Superior en ejercicio, para tributar a un desempeño profesional competente, ético y comprometido. Ello exige que las actividades formativas, por una parte, deben ser coherentes con las políticas educativas universitarias, los programas de estudio que imparte el profesorado participante y estar centradas en un aprendizaje activo y autorregulado del estudiantado; y, por otra parte, deben potenciar la reflexión crítica de los problemas de la práctica educativa (incluida la autorreflexión) y su solución, a partir de la experiencia individual y colectiva de los presentes. Además, mediante estas actividades formativas, el profesorado participante debe percibir los cambios que se pretenden introducir como significativos para ellos. Este enfoque podría conducir a entender mejor al profesorado, a analizar sus prácticas educativas y a proporcionarles un desarrollo profesional más significativo y efectivo. El trabajo metodológico individual y colectivo, que desarrolla el grupo docente para garantizar el cumplimiento de las demandas a partir de la preparación adecuada de sus clases, se considera una importante actividad de formación continua. 
Aunque este estudio está limitado por el pequeño tamaño de la muestra, proporciona información valiosa para el diseño de una estrategia de formación continua dirigida a la preparación del profesorado para darle cumplimiento, mediante su práctica educativa, a las demandas educativas de la sociedad del conocimiento:

- Se observa que el profesorado encuestado no reconoce en sí mismo necesidades formativas relacionadas con las demandas educativas de la sociedad del siglo XXI (ni con las cognitivo-instrumentales, ni con las axiológicas). Sin embargo, en general, no son capaces de explicitar metodologías apropiadas para su cumplimiento mediante la práctica educativa. Esta inconsistencia en las respuestas revela carencias de formación psicopedagógica para enfrentar estos desafíos que le plantea al profesorado la sociedad actual.

- A pesar de que las respuestas de las personas encuestadas muestran voluntad por contribuir al aprendizaje autorregulado a partir de la práctica educativa y las políticas educativas universitarias vigentes, refuerzan la importancia de que el estudiantado aprenda a autorregular su aprendizaje mediante una enseñanza sistemática e intencionada, pocos estudiantes muestran preparación para desempeñar un aprendizaje autónomo, y el hecho de que la población encuestada no sean capaz de ejemplificar cómo lo logran en su práctica educativa es una evidencia de que existen carencias formativas del profesorado en este aspecto. Esto se debe, en opinión de los investigadores, a la complejidad que supone la aprehensión del cambio de una concepción tradicional del proceso docente educativo hacia las concepciones que subyacen a un aprendizaje autorregulado. Ese cambio de mentalidad en el profesorado, a nivel consciente, y su influencia en la práctica educativa, supone un riguroso trabajo de formación del profesorado en la propia institución universitaria.

- Aunque en las respuestas al cuestionario no se revelaron grandes necesidades formativas en relación con el empleo de las TIC, esta temática es obligatoria en la formación continua, puesto que ella evoluciona rápidamente y cada día aparecen variadas innovaciones con aplicación al sistema educativo, lo que exige la actualización permanente de docentes para un desempeño profesional de alta calidad. La escasez formativa del profesorado en TIC implica una escasa o nula respuesta a las demandas sociales.

- Las respuestas de los encuestados y las encuestadas revelan carencias importantes en el trabajo metodológico. Los resultados están alejados de las potencialidades del trabajo 
metodológico como parte de la formación continua, dada su influencia decisiva en el desempeño profesional y en el desarrollo motivacional y humano de los implicados, así como en la formación de un profesorado reflexivo.

- La Universidad de Artemisa no cuenta con una oferta dinámica de formación continua que pueda involucrar a todo su personal docente y que responda a las necesidades formativas del profesorado.

En cuanto a los alcances y limitaciones de este estudio se debe señalar que el empleo de un solo instrumento, como el aplicado, aun cuando valora las necesidades formativas en todas las demandas educativas identificadas, no constituye el único mecanismo de identificación de necesidades formativas en relación con las demandas educativas de la sociedad del conocimiento. El instrumento por sí mismo representa tan solo la primera etapa de lo que deberá de ser todo el proceso de identificación de necesidades de formación continua. Este proceso incluirá las políticas universitarias para el período electivo a nivel internacional en el país y en la Universidad de Artemisa, los informes de resultados del trabajo metodológico del período anterior en la Universidad, los resultados de las evaluaciones anuales y de los controles a clases realizados al profesorado en el período anterior, entre otros.

\section{Referencias}

Al-Majed, Asma., Al-Kathiri, Fátima., Al-Ajmi, Sara., y Al-Hamlan, Suad. (2017). 21st Century Professional Skill Training Programs for Faculty Members-A Comparative Study between Virginia Tec University, American University y King Saud University. Higher Education Studies; 7(3), 122-131. doi: http://doi.org/10.5539/hes.v7n3p122

Almarshad, Yousef. (2017). Training Needs for the Professional Development of Social-Studies Teachers at the Intermediate Stage in Al-Jouf in Light of Modern Teaching Trends: A Field Study. Journal of Education and Learning, 6(2), 171-186. doi: http://dx.doi.org/10.5539/jel.v6n2p171

Barber, Michael y Mourshed, Mona (2008). Cómo hicieron los sistemas educativos con mejor desempeño del mundo para alcanzar sus objetivos. Recuperado de http://eduteka.icesi.edu.co/articulos/InformeMcKensey

Bayar, Adem., y Kösterelioğlu, İlker. (2014). Satisfaction levels of teachers in professional development activities in Turkey. Turkish Studies, International Periodical For the Languages, Literature and History of Turkish or Turkic, 9(2), 321-333. Recuperado de https://www.academia.edu/11872837/SATISFACTION LEVELS OF TEACHERS IN PROFESSIONAL DEVELOPMENT ACTIVITIES IN TURKEY 
Borko, Hilda., Jacobs, Jennifer., y Koellner, Karen. (2010). Contemporary approaches to teacher professional development. In Penelope Peterson, Eva Baker, Barry McGaw, (Eds.), International Encyclopedia of Education, (Vol. 7, pp. 548-556). Oxford: Elsevier. https://doi.org/10.1016/B978-0-08-044894-7.00654-0

Bozpolat, Ebru. (2016). Investigation of the Self-Regulated Learning Strategies of Students from the Faculty of Education Using Ordinal Logistic Regression Analysis. Educational Sciences: Theory \& Practice, 16(1), 301-318. doi: http://doi.org/10.12738/estp.2016.1.0281

Cabero Almenara, Julio. (2003). Replanteando la Tecnología Educativa. Comunicar, Revista Iberoamericana de Comunicación y Educación, (21), 22-30. Recuperado de https://www.revistacomunicar.com/pdf/comunicar21.pdf

Castellanos Simons, Doris., Castellanos Simons, Beatriz., Llivina Lavigne, Miguel J., Silverio Gómez, Mercedes., Reinoso Cápiro, Carmen., García Sánchez, Celina. (2007). Aprender y enseñar en la escuela: una concepción desarrolladora. La Habana, Cuba: Editorial Pueblo y Educación.

Colorado-Aguilar, Brenda., y Edel-Navarro, Rubén. (2015). La usabilidad de TIC en la práctica educativa. RED, Revista de Educación a Distancia, No. 30. Recuperado de http://www.um.es/ead/red/30/edel.pdf

Cuevas Jiménez, Adrián. (2016). La educación superior ante los desafíos sociales. Alteridad. Revista de Educación, 11(1), 101-109. doi: http://doi.org/10.17163/alt.v11n1.2016.08

Duffy, Gerald. (1994). Professional development schools and the disempowerment of teachers and professors. Phi Delta Kappan, 75(8), 596-600. Recuperado de https://www.jstor.org/stable/20405182?seq=1

Estrada García, Aurora. (2016). Instrumento para detectar las necesidades de formación docente. Revista Iberoamericana de Producción Académica y Gestión Educativa, 3(6). Recuperado de https://www.pag.org.mx/index.php/PAG/article/download/618/709

Fernández Aquinol, Orlando., Valdés Puentes, Roberto., González Carmenate, Maricela. (2008). Formación docente y trabajo metodológico en la universidad: resultados de una experiencia de desarrollo profesional centrada en la reflexión, el apoyo mutuo y la supervisión. Pro-Posições, v. 19, n. 1 (55) - jan./abr. doi: https://doi.org/10.1590/S0103$\underline{73072008000100013}$

Goel, Chanchal. (2019). Improving professional practices of in service teachers in Delhi. International Online Journal of Education and Teaching (IOJET), 6(3), 432-441. Recuperado de http://iojet.org/index.php/IOJET/article/view/672

González Tirado, Rosa M., y Gonzáles Maura, Viviana. (2007). Diagnóstico de necesidades y estrategias de formación docente en las universidades. Revista Iberoamericana de Educación, (43) $1-14$. Recuperado de https://rieoei.org/historico/deloslectores/1889Maura.pdf 
Hernández Hurtado, Juan. (2014). Diseño y construcción de un laboratorio de estadística descriptiva e inferencial, probabilidades y diseño experimental, para el aprendizaje autónomo en ciencias básicas e ingeniería mediado por ambientes virtuales de aprendizaje (Tesis de Maestría). Recuperado de https://silo.tips/download/laboratoriovirtual-de-estadistica-pagina-1-de-56

Hernández Sampieri, Roberto., Fernández Collado, Carlos., y Baptista Lucio, Pilar. (2014). Metodología de la Investigación. México: McGraw-Hill / Interamericana Editores, S.A. de C.V.

Horruitiner, Pedro. (2006). La Universidad Cubana: el modelo de formación. La Habana: Editorial Félix Varela.

Kabadayi, Abdülkadir. (2016). A Suggested In-service Training Model Based on Turkish Preschool Teachers' Conceptions for Sustainable Development. Journal of Teacher Education for Sustainability, 18(1), 5-15.doi: https://doi.org/10.1515/jtes-2016-0001

López, Ariadna Isabel., García, Omar., Pérez, Ricardo., Montero, Virginia. y Rojas, Elsa Liliana. (2016). Los Profesores de Tiempo Parcial en las universidades públicas estatales: una profesionalización inconclusa. Revista de la Educación Superior, 45(180), 23-29. doi: http://dx.doi.org/10.1016/j.resu.2016.06.007

Marcelo, Carlos. (2001). Aprender a enseñar para la Sociedad del Conocimiento. Revista Complutense de Educación, 12(2), 531-593. Recuperado de https://revistas.ucm.es/index.php/RCED/article/download/RCED0101220531A/16749

Ministerio de Educación Superior, Cuba. (2018). Reglamento de Trabajo Docente y Metodológico. Resolución Ministerial 2/2018. La Habana, Cuba: MES.

Ministerio de Educación Superior, Cuba. (2016). Documento base para el diseño de los planes de estudio "E". La Habana, Cuba: MES.

Montes, Diana A. y Suárez, Cecilia I. (2016). La formación docente universitaria: claves formativas de universidades españolas. Revista Electrónica de Investigación Educativa, 18(3), 51-64. Recuperado de http://redie.uabc.mx/redie/article/view/996

Núñez, José C., Solano, Paula., González-Pienda, Julio A., Rosário, Pedro. (2006). El aprendizaje autorregulado como medio y meta de la educación. Papeles del Psicólogo, 27(3), 139-146. Recuperado de http://www.redalyc.org/articulo.oa?id=77827303

Öztürk, Mustafa. (2019). An Evaluation of an Innovative In-Service Teacher Training Model in Turkey. International Journal of Higher Education, 8(1), 23-36. doi: https://doi.org/10.5430/ijhe.v8n1p23

Pérez Jacinto, Alipio Omar. (febrero de 2017). Los modelos estadísticos en los sistemas y proceso complejos de la realidad educativa. Curso pre congreso impartido en el Congreso Internacional Pedagogía, La Habana, Cuba.

Quintero Tapia, John J., Miranda Jaña, Christian E., y Rivera Rivera, Pablo J. (2018). Tendencias de investigación en formación permanente de profesores: estado del arte e 
interpretación de actores clave. Revista Electrónica Actualidades Investigativas en Educación, 18(2), 1-29. doi: https://doi.org/10.15517/aie.v18i2.33174

Rodríguez Jiménez, Andrés., Delgado Yanes, Nilda., Charbonet Martell, Miguel E., Velázquez, Arianni., Fundora Ramírez, Patricia., Ortiz Azahada, Arays., Reyes Cárdenas, Yaima., Baldriche, René., Hernández Cruz, Yoel., Villamil Galán, Lucila., Pedroso, Dorna., Horta Castro, Zuraima. (2019). El modelo pedagógico: La meta y los principios como componentes. Caracterización del proceso de enseñanza-aprendizaje a partir de las demandas educativas de la sociedad cubana del siglo XXI (Informe de resultado investigativo). Artemisa, Cuba: Universidad de Artemisa, Centro de Estudios de Educación y Desarrollo.

Rodríguez, Andrés., y Miqueli, Bertha. (2019). La estructura de la variable proceso de formación de profesionales en pregrado. Transformación, 15(1), 107-125. Recuperado de http://scielo.sld.cu/scielo.php?script=sci arttext\&pid=S2077-29552019000100110

Sakkoulis, Dimitris P., Asimaki, Anna. y Vergidis, Dimitris. (2018). In-service Training as a Factor in the Formation of the Teacher's Individual Theory of Education. International Education Studies; 11(3), 48-60. doi: https://doi.org/10.5539/ies.v11n3p48

Solak, Erkan. y Çer, Ekrem. (2018). Examining High-performing Education Systems in Terms of Teacher Training: Lessons Learnt for Low-performers. Journal of Curriculum and Teaching, 7(1), 42-51. doi: https://doi.org/10.5430/jct.v7n1p42

Texidor Pellón, Raisa., Reyes Miranda, Daniel., Berry González, Soraya., Cisnero Reyna, Caridad. (2017). Las Tecnologías de la Información y la Comunicación en la enseñanza de inglés en Ciencias Médicas. Educación Médica Superior, 31(2), 1-8. Recuperado de http://scielo.sld.cu/pdf/ems/v31n2/ems19217.pdf

Valle Lima, Alberto. (2012). La investigación pedagógica. Otra mirada. La Habana: Ministerio de Educación Cuba, Instituto Central de Ciencias Pedagógicas.

Yolcu, Hüseyin. y Kartal, Sadik. (2017). Evaluating of In-service Training Activities for Teachers in Turkey: A Critical Analysis. Universal Journal of Educational Research 5(6), 918-926. doi: https://doi.org/10.13189/ujer.2017.050602

Ulla, Mark B. (2018). In-service Teachers' Training: The Case of University Teachers in Yangon, Myanmar. Australian Journal of Teacher Education, 43(1), 66-77. Recuperado de: http://ro.ecu.edu.au/ajte/vol43/iss1/4

Zimmerman, Barry J. (2008). Investigating self-regulation and motivation: Historical background, methodological developments, and future prospects. American Educational Research Journal, 45(1), 166-183. doi: http://doi.org/10.3102/0002831207312909 
Revista indizada en

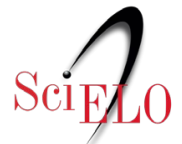
redalyc latindex

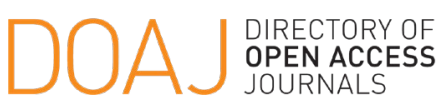

Distribuida en las bases de datos:

- Dialnet

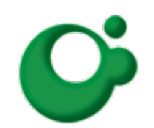
SHERPA/RøMEO REDIB

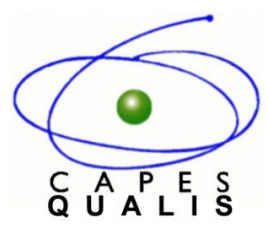

MIAR 